\title{
MicroRNA-802 increases hepatic oxidative stress and induces insulin resistance in high-fat fed mice
}

\author{
XI YANG $^{1 *}$, HANYING XING $^{2 *}$, JINGZHEN LIU $^{1}$, LINQUAN YANG $^{2}$, HUAN MA $^{2}$ and HUIJUAN MA ${ }^{1,2}$ \\ ${ }^{1}$ Department of Endocrinology; ${ }^{2}$ Hebei Key Laboratory of Metabolic Disease, \\ Hebei General Hospital, Shijiazhuang, Hebei 050051, P.R. China
}

Received August 30, 2018; Accepted March 29, 2019

DOI: $10.3892 / \mathrm{mmr} .2019 .10347$

\begin{abstract}
The expression of microRNA-802 (miR-802) is known to be associated with insulin resistance (IR); however, the mechanism remains unclear. The present study investigated how miR-802 contributes to the development of IR using C57BL/6J mice fed a high-fat diet (HFD) to establish a model of IR. Adeno-associated virus overexpressing miR-802 was administered to the mice via tail vein injection. The effects of miR-802 on reactive oxygen species (ROS), lipid peroxidation (LPO) and the activities of multiple ROS-related enzymes were investigated. Western blot analysis was used to estimate the protein levels of extracellular signal regulated kinase (ERK), p38mitogen-activated protein kinases (p38MAPK), c-Jun N-terminal kinase (JNK), insulin receptor substrate 1 (IRS-1) and protein kinase $\mathrm{B}$ (AKT1). The results demonstrated that the levels of ROS and LPO production were increased in the livers of the miR-802-treated group compared with the control group. The activities of the ROS-related enzymes were reduced. Furthermore, the expression of phosphorylated (phosphor)-p38MAPK and phosphor-JNK were upregulated in the miR-802 overexpression group, whereas there was no difference in the expression levels of phosphor-ERK. The expression levels of phosphor-AKT1 were reduced in the miR-802-treated group and these effects were reversed by miR-802 knockdown. In conclusion, the results demonstrate that miR-802 may cause IR by activating the JNK and p38MAPK pathways to increase hepatic oxidative stress.
\end{abstract}

Correspondence to: Dr Huijuan Ma, Department of Endocrinology, Hebei General Hospital, 348 He Ping West Road, Shijiazhuang, Hebei 050051, P.R. China

E-mail: huijuanma76@163.com

*Contributed equally

Key words: insulin resistance, oxidative stress, microRNA-802, p38mitogen-activated protein kinases, c-Jun $\mathrm{N}$-terminal kinase

\section{Introduction}

Insulin resistance (IR) is a condition in which the body does not respond appropriately to circulating insulin. It is common in various metabolic disorders, such as diabetes, obesity, hypertension and coronary heart disease. IR occurs in a number of tissues, including liver, muscle and fat (1).

As a newly identified class of small non-coding RNA, microRNA (miRNA/miR) have been widely implicated in numerous physiological processes, including the pathogenesis of diabetes and obesity (2). miRNAs regulate the translation of mRNAs by specifically binding to the 3'-untranslated region of mRNA and inhibiting translation or targeting the transcript for degradation (3). Previous studies have suggested that dysregulation of hepatic miRNAs leads to type 2 diabetes mellitus (T2DM). A number of miRNAs have been reported to contribute to IR in vivo (4-6).

MicroRNA-802 (miR-802) expression has been reported at high levels in the serum and livers of C57BL/6J mice fed a high-fat high-sucrose diet (7), db/db mice $\left(\operatorname{Lepr}^{\mathrm{db} / \mathrm{db}}\right)$ and obese subjects (8). Overexpression of miR-802 can cause IR and impair glucose tolerance, whereas downregulated miR-802 expression in obese mice improved these metabolic parameters, suggesting that higher miR-802 expression results in an increased risk of obesity-associated IR (8). miR-802 not only induces IR, but also enhances both basal and forskolin-induced expression of glucose-6-phosphatase (8). However, the roles of miR-802 in oxidative stress of the liver remain to be elucidated. It is known that diabetic complications in target organs result from chronic elevations in the levels of glucose. The pathogenic effect of high glucose, possibly in combination with fatty acids, is mediated to a significant extent via increased production of reactive oxygen species (ROS) and the subsequent oxidative stress. An increase in the levels of insulin, free fatty acid and/or glucose can elevate ROS production and oxidative stress, as well as activate stress-sensitive pathways. $\beta$-cells are sensitive to ROS, which may be associated with their lack of relevant free-radical quenching (antioxidant) enzymes such as catalase (CAT), glutathione peroxidase (GSH-Px) and superoxide dismutase (SOD) (9). Indeed, persistent ROS generation can diminish insulin action through activation of serine-threonine kinase cascades that, in turn, phosphorylate several targets, including the insulin receptor and the insulin receptor substrate (IRS) proteins, with consequent decrease 
in insulin-stimulated tyrosine phosphorylation, thereby increasing IR and accelerating the progression to overt type 2 diabetes (10).

In the present study, a model of IR was established by feeding mice a high-fat diet (HFD). An adeno-associated virus (AAV) overexpressing miR-802 was administered to the mice via tail vein injection. AAV vectors are single-stranded DNA viruses and one of the smallest types of vector, with a packaging limit of $\sim 5 \mathrm{~kb}$. AAV vectors stably and efficiently infect a wide variety of dividing or quiescent cells and have been clinically studied in multiple tissue types (11). In particular, AAV vectors provide efficient systemic gene delivery directly to skeletal muscle in vivo and lentivirus vectors may enable a combination of ex vivo gene modification strategies with cell-mediated therapies. AAVs can deliver substantially higher miRNA copy numbers per cell than lentiviruses (hundreds vs. a single or few copies) and therefore provide greater in vivo efficacy and a wider therapeutic window (12). Therefore, AAVs and not lentiviruses were used in the present study. Subsequently, it was investigated whether miR-802 induces IR in the mice by increasing hepatic oxidative stress.

\section{Materials and methods}

Animal study. Male C57BL/6J mice ( $\mathrm{n}=50$; age, 5 weeks old; weight, 16-19 g) were obtained from Charles River Laboratories, Inc. (Beijing, China). The animals were housed at a constant temperature $\left(22 \pm 2^{\circ} \mathrm{C}\right)$ and humidity $(50 \pm 10 \%)$ under a 12-h light/dark cycle with access to a standard chow diet and water ad libitum. Following 1 week of acclimatization, the 6-week old mice were fed either a normal diet (CON group; $n=10$ ) or high fat diet (HFD group; $n=40$ ) for the next 12 weeks. The high fat diet consisted of $60 \% \mathrm{kcal}$ fat, $20 \%$ kcal carbohydrate and $20 \%$ kcal protein (cat. no. D12492; Huafukang Biology Co., Ltd.). The normal diet consists of $10 \% \mathrm{kcal}$ fat, $70 \% \mathrm{kcal}$ carbohydrate and $20 \% \mathrm{kcal}$ protein (cat. no. D12450J; Huafukang Biology Co., Ltd.). The present study was approved by the Animal Care and Use Committee of Hebei General Hospital (Shijiazhuang, China).

Recombinant AAVs expressing miR-802 (AAV-miR-802, 5'-GGUCCUAUUAUUUGCAAUCAGUAACAAAGAUUC AUCCUUGUGUCAAUCAUACAACACGGAGAGUCU UUGUCACUCAGUGUAAUUAAUAGCCUUCACC-3') or miR-802 sponges (inhibitor; AAV-miR-802-SP, 3'-AAGGAT GAATCTTTGTTACTGATATACAAGGATGAATCTTTG TTACTGAACATCAAGGATGAATCTTTGTTACTGATC TTCAAAGGATGAATCTTTGTTACTGA-5') and a negative control AAV vector containing green fluorescent protein (AAV-GFP) were purchased from Shanghai HanHeng Co., (Shanghai, China).

After 12 weeks of the HFD, the AAV vectors were administered to the mice via tail vein injection at a dose of $1 \times 10^{11}$ plaque-forming units in $0.1 \mathrm{ml}$ PBS. The HFD group $(n=40)$ were subdivided randomly into the following four groups (n=10 mice/group): HFD, HFD + AAV-miR-802, HFD + AAV-GFP and HFD + AAV-miR-802-SP. After injection, mice were fed with a HFD for 5 weeks. The mice were sacrificed after AAV injection 5 weeks via cervical dislocation. The liver was quickly removed after sacrifice and then divided into 5 parts for further study. One part was washed with cold phosphate-buffered saline immediately and embedded in OCT embedding agent for dihydroethidium (DHE)-staining. The rest of the liver tissue was frozen at $-80^{\circ} \mathrm{C}$ in an EP tube in liquid nitrogen respectively for the detection by western blotting, triglyceride (TG), lipid peroxidation (LPO) and ROS-related enzyme activities. Ensure that detections are completed as soon as possible and prevent repeated freezing and thawing of the tissue. The time line for the experiment is depicted in Fig. 1.

Sample collection and total cholesterol (TC) and TG measurement. Following the experimental period, the mice were fasted overnight and then sacrificed. All samples were immediately placed in liquid nitrogen after removal in order to minimize damage. The blood plasma and liver were sampled from the same mice. The levels of glucose, insulin, total TC (A111-1; Nanjing Jiancheng Bioengineering Institute, Nanjing, China) and TG (A110-1; Nanjing Jiancheng Bioengineering Institute, Nanjing, China) in the blood were measured.

Enzyme kits were used to measure the hepatic TG (A110-1; Nanjing Jiancheng Bioengineering Institute, Nanjing, China), according to the manufacturer's protocol. The TG content of the livers was measured by first weighing the liver samples and then homogenizing them with anhydrous ethanol $(1 \mathrm{~g}$ : $9 \mathrm{ml})$. The samples were centrifuged at room temperature for $10 \mathrm{~min}$ at 2,500 rpm $(1,048.1 \mathrm{x} \mathrm{g})$ and then the supernatant was removed to determine the level of TG. The supernatants were mixed with reagent from kit according to the manufacturer's protocol. The reaction mixtures were placed in a water bath at $37^{\circ} \mathrm{C}$ for $10 \mathrm{~min}$. The absorbance was measured at $510 \mathrm{~nm}$. The results were expressed as $\mathrm{mmol} / \mathrm{g}$ protein.

Intraperitoneal glucose tolerance test (IPGTT) and serum insulin analysis. IPGTT were carried out at 12 weeks after HFD intervention (18 weeks of age) and at 4 weeks after AAV injection (22 weeks of age). The IPGTT was performed via intraperitoneal injection of glucose $(2 \mathrm{~g} / \mathrm{kg})$ after fasting overnight (13). Blood was collected from the tail vein of the mice in a heparinized capillary tube and the blood glucose was analyzed with a glucometer (ACCU-CHEK; Roche Diagnostics GmbH, Mannheim, Germany) at $0 \mathrm{~min}$ (prior to the glucose load), and at 15, 30, 60 and $120 \mathrm{~min}$ after the glucose load. The levels of insulin were measured using insulin ELISA kits (ALCO Chem. Inc.; cat. no. 04505) according to the manufacturers' protocol.

IR was calculated using the QUICKI method: QUICKI $\left.=1 /\left[\log \left(\mathrm{I}_{0}\right)+\log \left(\mathrm{G}_{0}\right)\right]\right)$, where $\mathrm{I}_{0}$ is the fasting insulin level $(\mu \mathrm{U} / \mathrm{ml})$ and $\mathrm{G}_{0}$ is the fasting blood glucose level (mg/dl) (14). The QUICKI method is a mathematical model that correlates well with the 'gold standard' in IR assays, the euglycemic clamp (14) and mice or rats with IR tend to have lower QUICKI or insulin sensitivity values.

Measurement of ROS production. The levels of ROS were measured with DHE, which can bind irreversibly to DNA to produce ETH-DNA and displays red fluorescent signal when oxidized (15). The liver was removed after sacrifice, a part of the liver was taken and the unfixed tissue was embedded in OCT embedding agent to prepare frozen slices using the dry ice acetone method for cryosectioning. The method is described as follows: $200 \mathrm{ml}$ acetone was injected into a small wide-mouth thermos flask. Dry ice was gradually added to the 


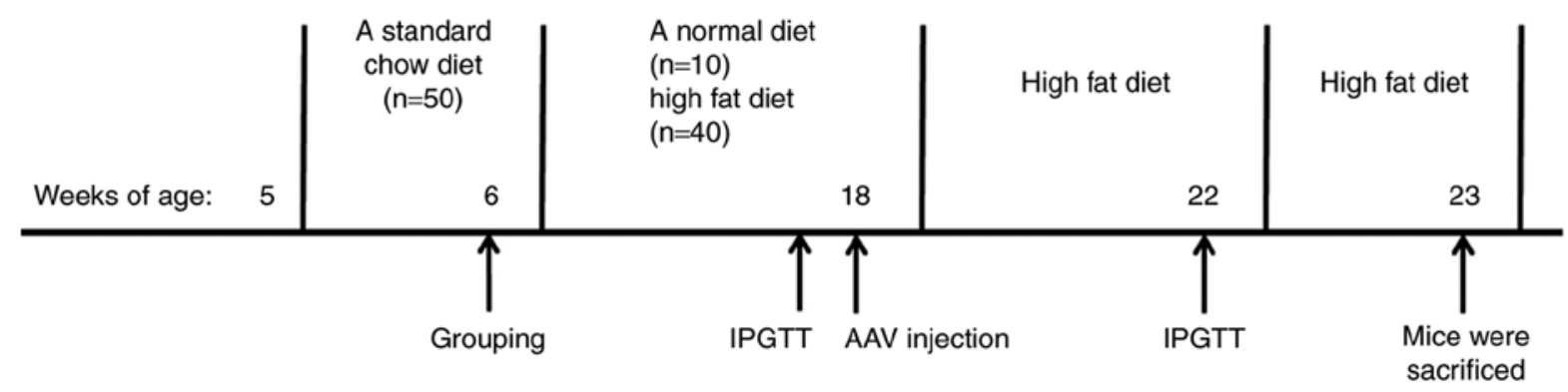

Figure 1. Study design timeline. At the beginning, all 5 week-old mice were fed with a standard chow diet for 1 week, then fed either a normal diet or high fat diet for 12 weeks. At 18 weeks of age, the AAV vectors were administered to the mice. IPGTT were carried out at 18 and 22 weeks. At the end of the experiment, mice were sacrificed at 23 weeks. AAV, Adeno-associated virus; IPGTT, intraperitoneal glucose tolerance test.

flask until it was saturated and did not bubble. The temperature was $\sim-70^{\circ} \mathrm{C}$. A small beaker containing $50 \mathrm{ml}$ isopentane was placed into a dry ice-acetone saturated solution. Tissue blocks embedded in OCT were then frozen at $-40^{\circ} \mathrm{C}$ in isopentane for $1 \mathrm{~min}$. The frozen segments were cut into $8-\mu \mathrm{m}$-thick transverse sections that were then placed on glass slides. Following incubation with DHE $(10 \mu \mathrm{m} / \mathrm{l})$ in a light-protected chamber at $37^{\circ} \mathrm{C}$ for $40 \mathrm{~min}$, ultrathin $(8 \mu \mathrm{m})$ frozen sections of the liver were prepared using a cryostat (Microm HM525; Thermo Fisher Scientific, Inc., Waltham, MA, USA) at $-20^{\circ} \mathrm{C}$. The fluorescence was measured using a fluorescent microscope (Leica DMI3000 B; Leica Microsystems GmbH, Wetzlar, Germany) at excitation/emission wavelengths of 530/616 nm.

Measurement of ROS-related enzyme activities and LPO. The liver samples were homogenized in ice-cold potassium phosphate-buffered saline at 10-20X dilution. Liver specimens $(10 \%)$ in $0.15 \mathrm{M} \mathrm{KCl}$ was prepared with a homogenizer from Pro Scientific, Inc. (PRO 200). After centrifuging at $1,257.7 \mathrm{x}$ g for $10 \mathrm{~min}$ at $4^{\circ} \mathrm{C}$, the tissue supernatants were used to measure the activity of the following anti-oxidant enzymes: CAT (cat. no. A007-1; Nanjing Jiancheng Biological Engineering Institute), SOD (cat. no. A001-3; Nanjing Jiancheng Biological Engineering Institute), reduced glutathione (GSH; cat. no. A006-1; Nanjing Jiancheng Biological Engineering Institute) and GSH-Px (cat. no. A005; Nanjing Jiancheng Biological Engineering Institute). All parameters were determined according to manufacturers' protocol.

The levels of LPO (cat. no. A106-1; Nanjing Jiancheng Biological Engineering Institute) were also measured. In brief, samples were mixed with reagent from kit. The reaction mixtures were placed in a $45^{\circ} \mathrm{C}$ water bath for $60 \mathrm{~min}$ and centrifuged at $1,677 \mathrm{x} \mathrm{g}$ at room temperature for $10 \mathrm{~min}$. The absorbance of the supernatant was measured at $586 \mathrm{~nm}$. The results were expressed as $\mu \mathrm{mol} / \mathrm{g}$ protein.

CAT activity was determined using a spectrophotometer at $405 \mathrm{~nm}$. In brief, samples were mixed with the reagent from the kit according to the manufacturer's protocol. A $0.5 \mathrm{~cm}$ optical path was used and zero adjustment to measure the absorbance. The amount of enzyme capable of catalyzing the degradation of $1 \mu \mathrm{mol}$ of $\mathrm{H}_{2} \mathrm{O}_{2}$ per min was defined as $1 \mathrm{U}$.

SOD activity was assayed by measuring its ability to inhibit the photoreduction of nitroblue tetrazolium (NBT) according to the manufacturer's protocol of the kit. The changes in absorbance at $450 \mathrm{~nm}$ were recorded after $37^{\circ} \mathrm{C}$ water bath for $20 \mathrm{~min}$. A total of 1 unit of SOD is defined as the amount that inhibits the NBT reaction by $50 \%$. Specific activity was defined as U/mg protein.

The tissue GSH concentrations were determined using a visible spectrum spectrophotometer. Briefly, fresh supernatant $(0.5 \mathrm{ml})$ was added to applied solution of reagent $1(2 \mathrm{ml})$ and centrifuged at $1,677 \mathrm{x} \mathrm{g}$ at room temperature for $10 \mathrm{~min}$. The mixture was rested for five $\mathrm{min}, 1 \mathrm{~cm}$ optical path was used and zero adjustment to measure the absorbance of each tube. The optical density was measured at $420 \mathrm{~nm}$ in the spectrophotometer.

Activity of GSH-Px was determined using a visible spectrum spectrophotometer according to the manufacturer's protocol. GSH-Px activity for tissues was expressed as U/mg protein.

Western blot analysis. Western blot analysis was conducted as previously described (16). To obtain total proteins, a part of liver tissue from the mice was lysed in a buffer containing $50 \mathrm{mM}$ TrisHCl (pH 7.4), $150 \mathrm{mM} \mathrm{NaCl}, 1 \%$ Triton X100, $0.1 \%$ SDS and $1 \mathrm{mM}$ phenylmethylsulfonyl fluoride. The protein concentration was measured using a bicinchoninic acid assay method (Beijing Solarbio Science and Technology, Co., Ltd., Beijing, China). Lysates of $1,015 \mu \mathrm{g}$ protein were separated by $10 \%$ SDSPAGE gel, transferred onto polyvinylidene difluoride membranes (EMD Millipore, Billerica, MA, USA), blocked with 5\% skimmed dry milk at room temperature for $1 \mathrm{~h}$ and probed with primary antibodies at $4^{\circ} \mathrm{C}$ overnight. The primary antibodies used included those for extracellular signal regulated kinase (ERK; CST4695; anti-rabbit; 1:1,000; Cell Signaling Technology, Inc.), phosphorylated (phosphor)-ERK (Thr202/Tyr204; CST4370; anti-rabbit; 1:2,000; Cell Signaling Technology, Inc.), p38mitogen-activated protein kinases (p38MAPK; CST8690; anti-rabbit; 1:1,000; Cell Signaling Technology, Inc.), phosphor-p38MAPK (Thr180/Tyr182; CST4511, anti-rabbit; 1:1,000; Cell Signaling Technology, Inc.), c-Jun N-terminal kinase (JNK; CST9252; anti-rabbit; 1:1,000; Cell Signaling Technology, Inc.), phosphor-JNK (Thr183/Tyr185; CST4671; anti-rabbit; 1:1,000; Cell Signaling Technology, Inc.), insulin receptor substrate-1 (IRS-1; CST3089; anti-rabbit; 1:1,000; Cell Signaling Technology, Inc.), phosphor-IRS-1 (Ser307; cat. no. ab3690; anti-rabbit; 1:2,000; Abcam), protein kinase B (AKT1; cat. no. CST75692; anti-rabbit, 
A
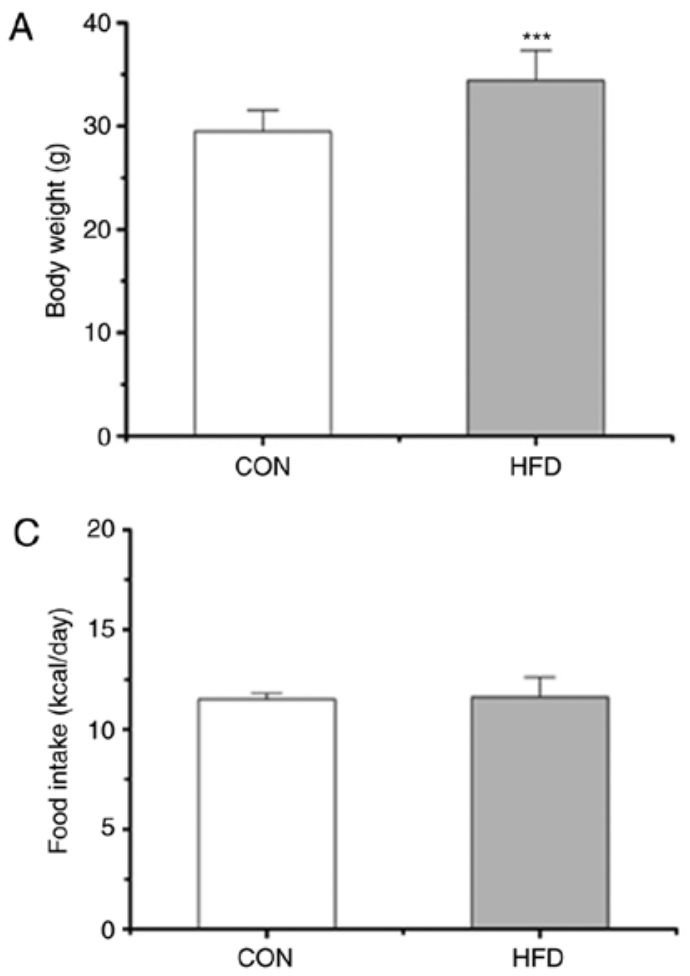

B
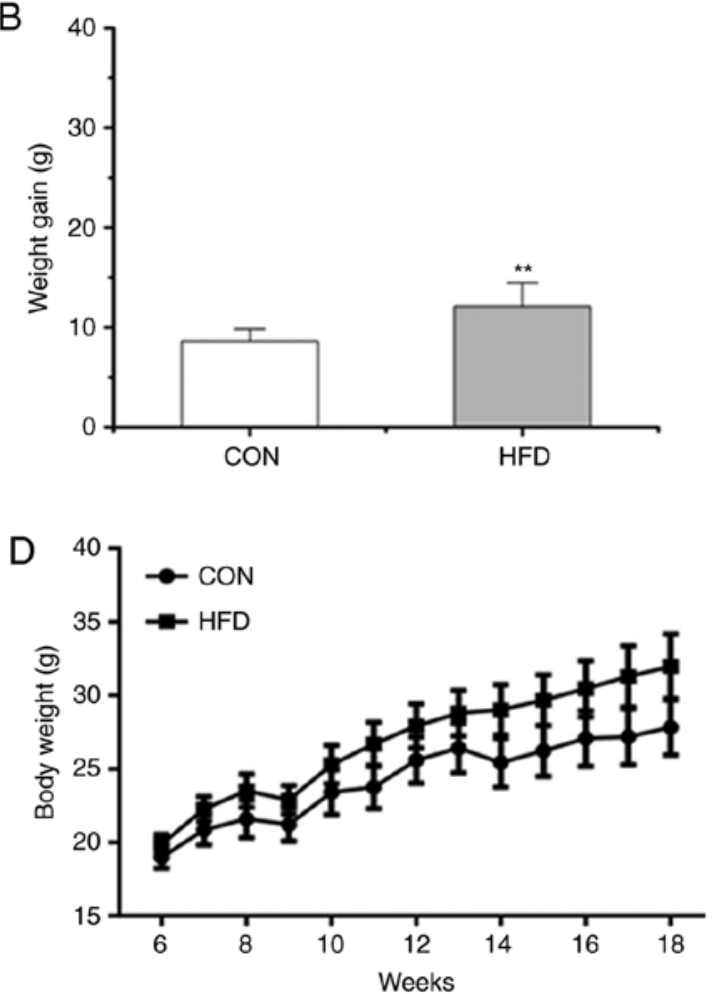

Figure 2. Analysis of physical parameters of the mice. Comparison of (A) body weight, (B) weight gain, (C) food intake and (D) body weight growth curves between the CON and HFD groups after 12 weeks of feeding. Data are presented as the mean \pm standard error of the mean $(\mathrm{n}=10)$. ${ }^{* * *} \mathrm{P}<0.01$ and ${ }^{* * * *} \mathrm{P}<0.001 \mathrm{vs}$. CON. CON, normal diet; HFD, high-fat diet.

1:1,000; Cell Signaling Technology, Inc.) and phosphor-AKT1 (Ser473; cat. no. CST9018; anti-rabbit, 1:1,000; Cell Signaling Technology, Inc.). The blots were subsequently incubated with horseradish peroxidaseconjugated secondary antibodies at room temperature for $1 \mathrm{~h}$, the secondary antibodies used were peroxidaseconjugated rabbit (cat. no. sc2031) or mouse (cat. no. sc2032) antibodies (1:10,000; both from GE Healthcare BioSciences, Pittsburgh, PA, USA) followed by detection via and ECL kit (cat. no. sc2048; Santa Cruz Biotechnology, Inc., Dallas, TX, USA). The protein bands were quantified by densitometry using ImageJ software (version 1.51k; National Institutes of Health, Bethesda, MD, USA). $\beta$-actin was used as a loading control (CST3700; antimouse; 1:10,000; Cell Signaling Technology, Inc.).

Statistical analysis. SPSS 17.0 software (SPSS, Inc., Chicago, IL, USA) was used for data analysis and the results were expressed as the mean \pm standard error of the mean. Student's ttest was performed for comparisons between two groups. A one-way analysis of variance was used to detect statistical significance followed by post hoc Dunn's test. The experiments were repeated three times. $\mathrm{P}<0.05$ was considered to indicate a statistically significant difference.

\section{Results}

Hepatic miR-802 expression increases in the HFD group. After diet intervention for 12 weeks, the body weight of the mice in the HFD group was increased compared with the CON group (Fig. 2A, B and D). Food intake was similar in the two groups (Fig. 2C). The levels of hepatic miR-802 expression were significantly increased in the HFD group compared with the $\mathrm{CON}$ group $(\mathrm{P}<0.001$; Fig. $3 \mathrm{~A})$.

After treatment for 5 weeks with the AAVs, the expression levels of miR-802 were highest in the HFD + AAV-miR-802 group and lowest in the HFD + AAV-miR-802-SP group (Fig. 3A). The body weight and food intake were not significantly different between the upregulated and downregulated miR-802 groups (Fig. 3B-D), which indicates that miR-802 has no significant influence on body weight.

Effect of miR-802 on blood glucose, serum insulin and IR. After intervention for 12 weeks with a HFD, the HFD mice exhibited a significant increase in the blood glucose and serum insulin levels compared to the CON group $(\mathrm{P}<0.001$; Fig. 4A-C). The IPGTT revealed that the HFD fed mice had higher blood glucose levels at 15 and 30 min compared with the CON group. The levels of blood glucose at 0,60 and $120 \mathrm{~min}$ were not significantly different between the two groups (Fig. 4A). After intervention for 5 weeks with AAV, in the mice with upregulated miR-802 expression, the blood glucose levels were further increased at 0,15 and 30 min than the HFD group. After administration of AAV-miR-802-SP, the elevated blood glucose levels were reduced at 0 min compared with the HFD group. The glucose levels at 15, 30, 60 and $120 \mathrm{~min}$ were not significantly different among the groups (Fig. 4D). The 5-week treatment with the miR-802 plasmid resulted in increased levels of blood glucose compared with the HFD group. The effect of AAV-miR-802-SP on the glucose levels was significantly reduced compared with the HFD group. 

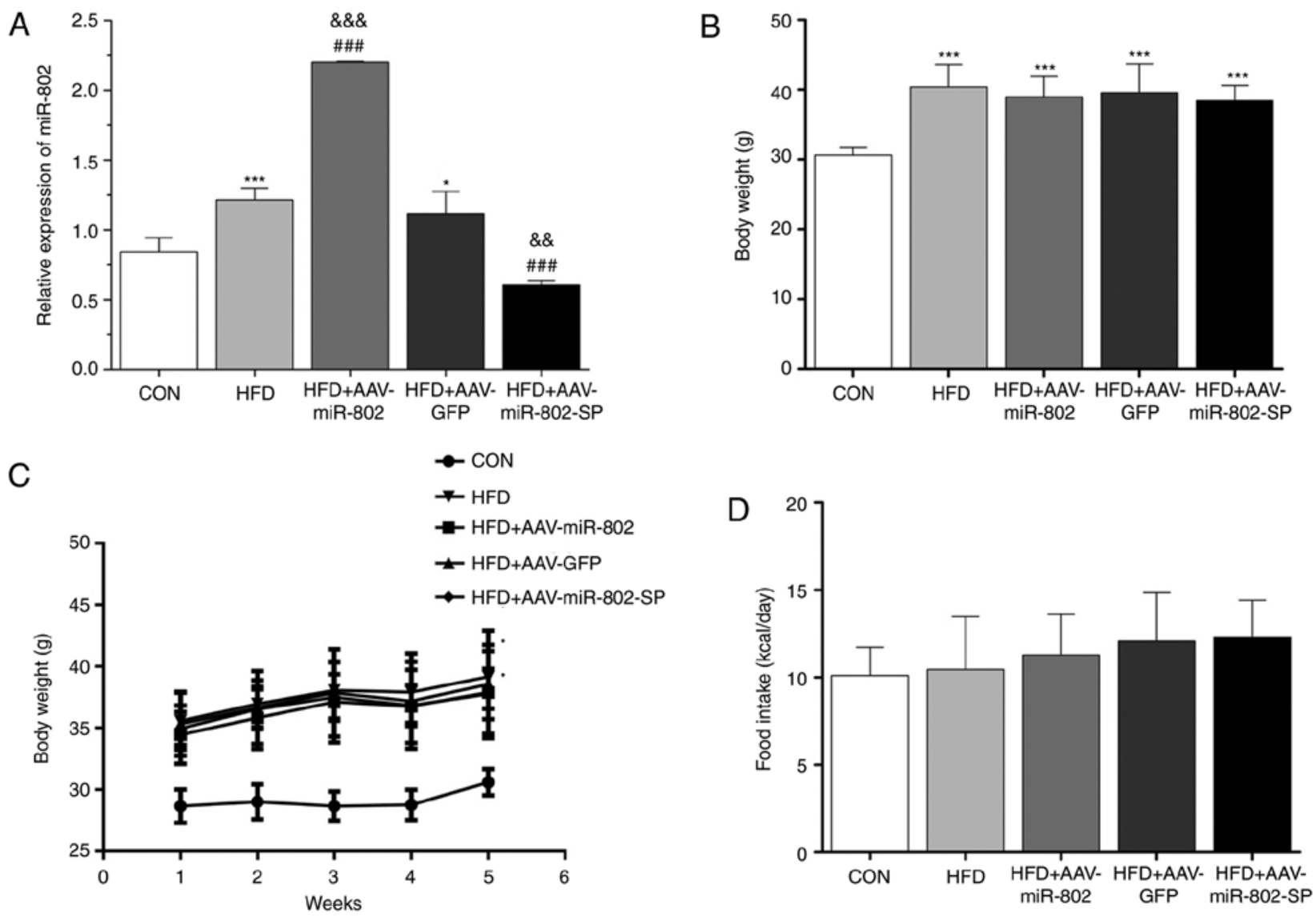

Figure 3. Analysis of miR expression and its association with body weight and food intake. (A) miR-802 expression levels were assayed with polymerase chain reaction in the liver. The effects of miR-802 on (B) body weight at 5 weeks and (C) trends over the course of the experiment and (D) food intake after treatment for 5 weeks. Data are presented as the mean \pm standard error of the mean $(\mathrm{n}=10) .{ }^{*} \mathrm{P}<0.05$ and ${ }^{* * * *} \mathrm{P}<0.001$ vs. CON; ${ }^{* \# \#} \mathrm{P}<0.001$ vs. HFD; and ${ }^{\& \&} \mathrm{P}<0.01$ and \&\&\&P $<0.001$ vs. HFD+AAV-GFP. miR-802, microRNA-802; CON, normal diet; HFD, high-fat diet; AAV-GFP, adeno-associated virus vector containing green fluorescent protein; AAV-miR-802, AAVs expressing miR-802; AAV-miR-802-SP, AAVs expressing miR-802 sponges.
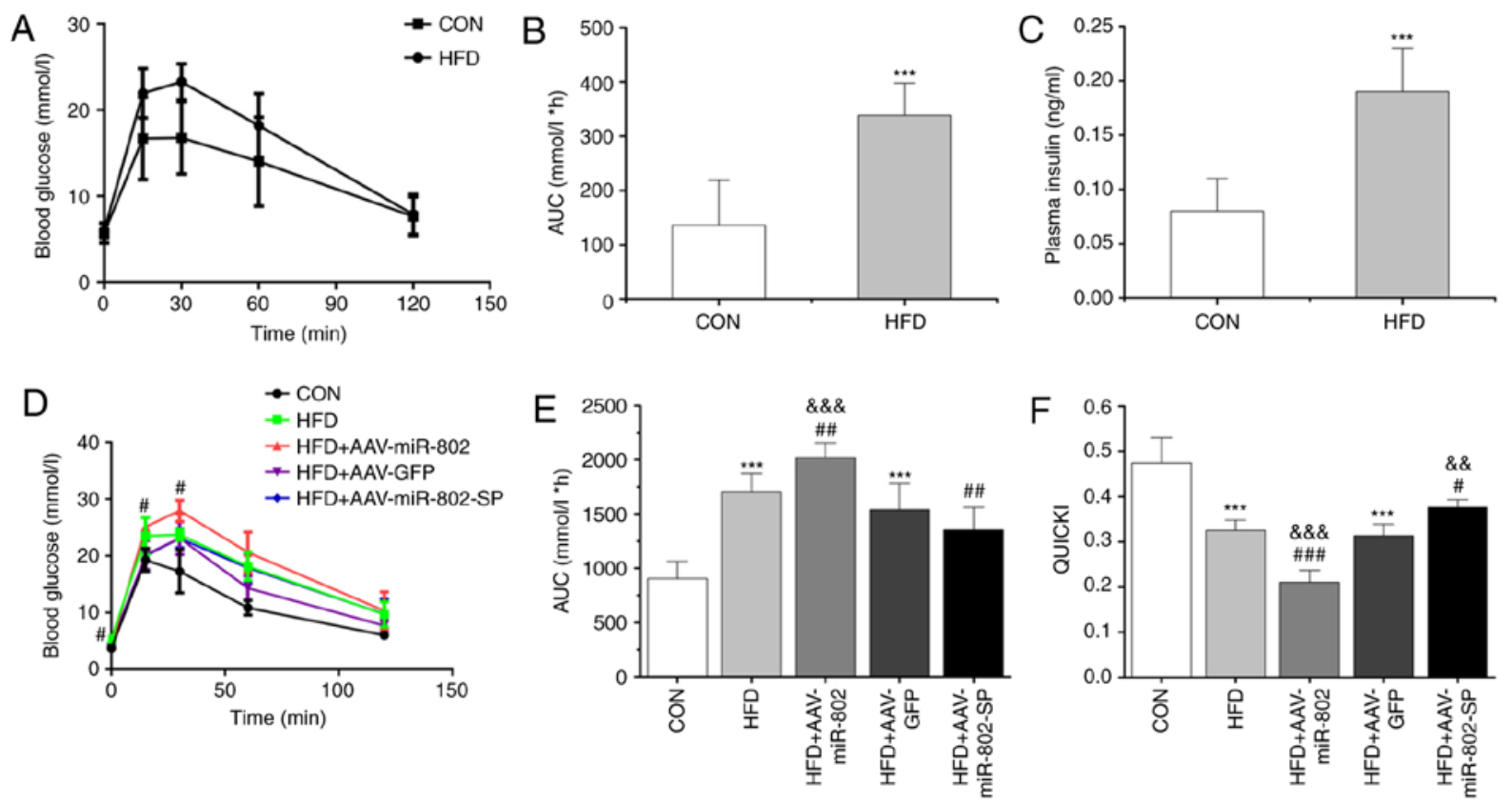

Figure 4. HFD induces whole-body glucose intolerance after 12 weeks of feeding. (A) The IPGTT was performed and (B) the AUC was calculated after 12 weeks of feeding. (C) The levels of insulin were compared between the groups. The effect of miR-802 on (D) the IPGTT, (E) AUC and (F) QUICKI after 5 weeks of miR-802 treatment. Data are presented as the mean \pm standard error of the mean $(\mathrm{n}=10) .{ }^{* * *} \mathrm{P}<0.001$ vs. CON; ${ }^{*} \mathrm{P}<0.05,{ }^{\# \#} \mathrm{P}<0.01$ and ${ }^{\# \# \# /} \mathrm{P}<0.001$ vs. HFD; and \&\& $\mathrm{P}<0.01$ and \&\&\& $<<0.001$ vs. HFD + AAV-GFP. HFD, high-fat diet; IPGTT, intraperitoneal glucose tolerance test; AUC, area under the curve; miR-802, microRNA-802; CON, normal diet; AAV-GFP, adeno-associated virus vector containing green fluorescent protein; AAV-miR-802, AAVs expressing miR-802; AAV-miR-802-SP, AAVs expressing miR-802 sponges. 

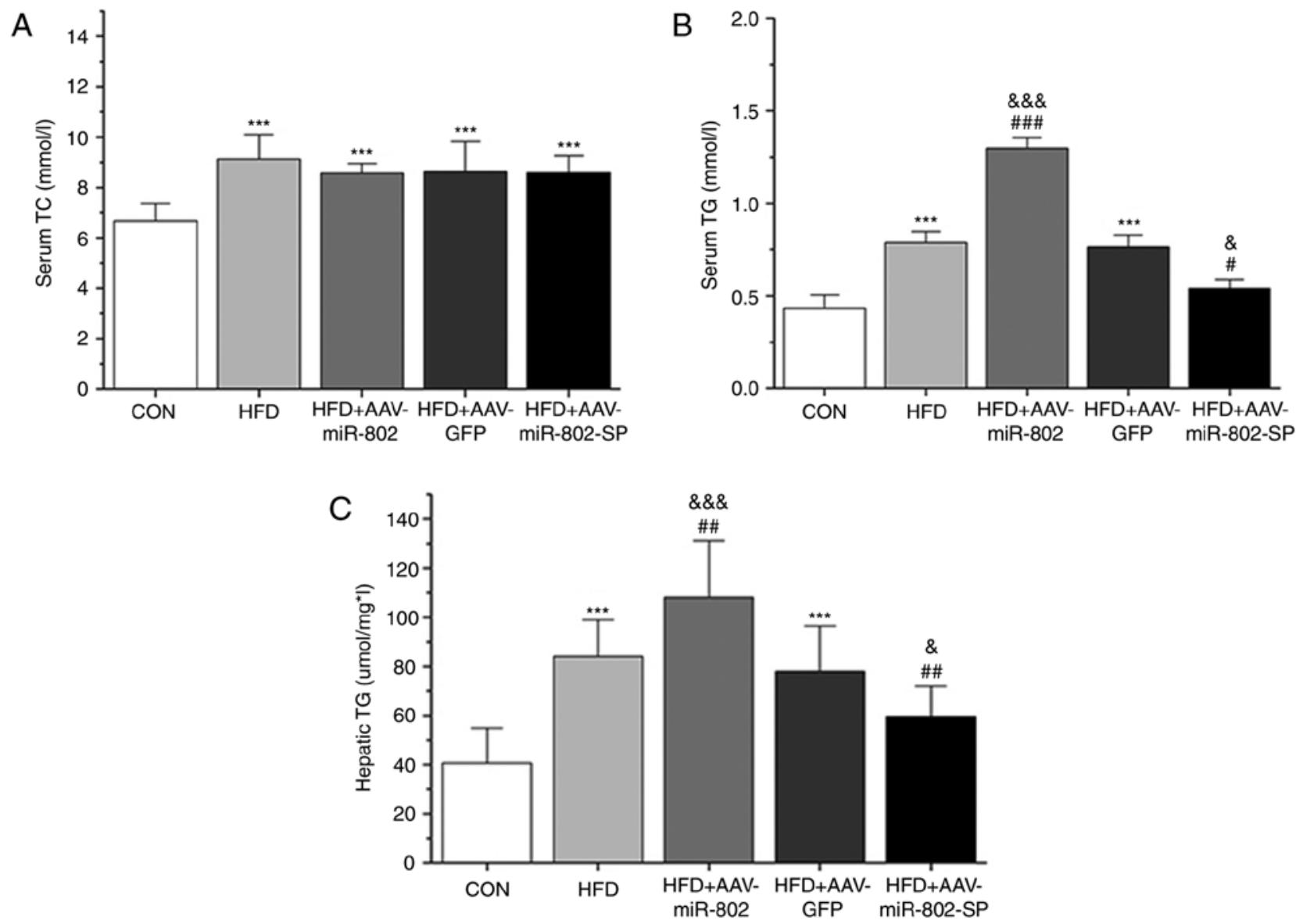

Figure 5. Analysis of biochemical parameters associated with a HFD. Effect of miR-802 on serum (A) TC and (B) TG, and (C) hepatic TG after 5 weeks of miR-802 treatment. Data are presented as the mean \pm standard error of the mean $(\mathrm{n}=10) .{ }^{* * * *} \mathrm{P}<0.001 \mathrm{vs}$. CON; ${ }^{\#} \mathrm{P}<0.05$; ${ }^{\# \#} \mathrm{P}<0.01$ and ${ }^{\# \# \#} \mathrm{P}<0.001 \mathrm{vs}$. HFD; and ${ }^{\&} \mathrm{P}<0.05$ and ${ }^{\& \& \&} \mathrm{P}<0.001$ vs. HFD + AAV-GFP. miR-802, microRNA-802; TC, total cholesterol; TG, triglycerides; CON, normal diet; HFD, high-fat diet; AAV-GFP, adeno-associated virus vector containing GFP; AAV-miR-802, AAVs expressing miR-802; AAV-miR-802-SP, AAVs expressing miR-802 sponges.

In agreement with these results, the area under the curve of the AAV-miR-802 group was significantly increased compared with those of the AAV-GFP and HFD groups $(\mathrm{P}<0.001)$. However, the difference between the AAV-miR-802-SP and AAV-GFP groups was not significant (Fig. 4E).

After 12 weeks on the HFD, the mice exhibited a significant reduction in the QUICKI values compared with the CON group $(\mathrm{P}<0.001$; Fig. $4 \mathrm{~F})$, which indicated that a mice model of IR was successfully established. miR-802 induced a further reduction in the QUICKI values compared with the HFD group (Fig. 4F), indicating that miR-802 exacerbates IR in the mice.

Effect of miR-802 on serum and liver lipid profiles. Serum TC and TG, and liver TG levels significantly were increased in the HFD group compared with the CON group $(\mathrm{P}<0.001$; Fig. 5A and B). AAV-miR-802 significantly increased the serum TG levels compared with the HFD group. AAV-miR-802 significantly increased the liver TG levels compared with the AAV-GFP and HFD groups, while AAV-miR-802-SP significantly reduced the levels of hepatic TG compared with the HFD group ( $\mathrm{P}<0.01$; Fig. 5C).

Effect of miR-802 on the activities of oxidative stress-related enzymes and antioxidant biomarkers in the liver. After
miR-802 treatment, the levels of LPO generation in the liver were significantly increased in the HFD group compared with the CON group $(\mathrm{P}<0.01)$. miR-802 significantly increased the levels of LPO compared with the AAV-GFP and HFD groups $(\mathrm{P}<0.01)$ and this effect was reversed by AAV-miR-802-SP (Fig. 6A).

The activities of the enzymes CAT, SOD and GSH-Px, as well as the GSH levels, were significantly reduced in the HFD group compared with the $\mathrm{CON}$ group $(\mathrm{P}<0.001)$. Overexpression of miR-802 further reduced the activities of these enzymes and the levels of GSH, and these effects were significantly reversed by AAV-miR-802-SP (P<0.05; Fig. 6B-E). Similarly, the levels of ROS generation in the liver were also increased after miR-802 treatment and AAV-miR-802-SP reversed this increase (Fig. 7). These results indicate that miR-802 increases hepatic oxidative stress.

Effect of miR-802 on the activities of the insulin signaling pathway in mouse livers. Insulin-stimulated glucose utilization relies on the activity of the insulin signaling pathway. Therefore, the activities of the insulin pathway-related components IRS-1 and AKT1 in the liver of the mice were determined.

After administration of miR-802 for 5 weeks, there were no significant changes in the protein expression levels of total IRS-1 and AKT1. The expression levels of phosphorylated 

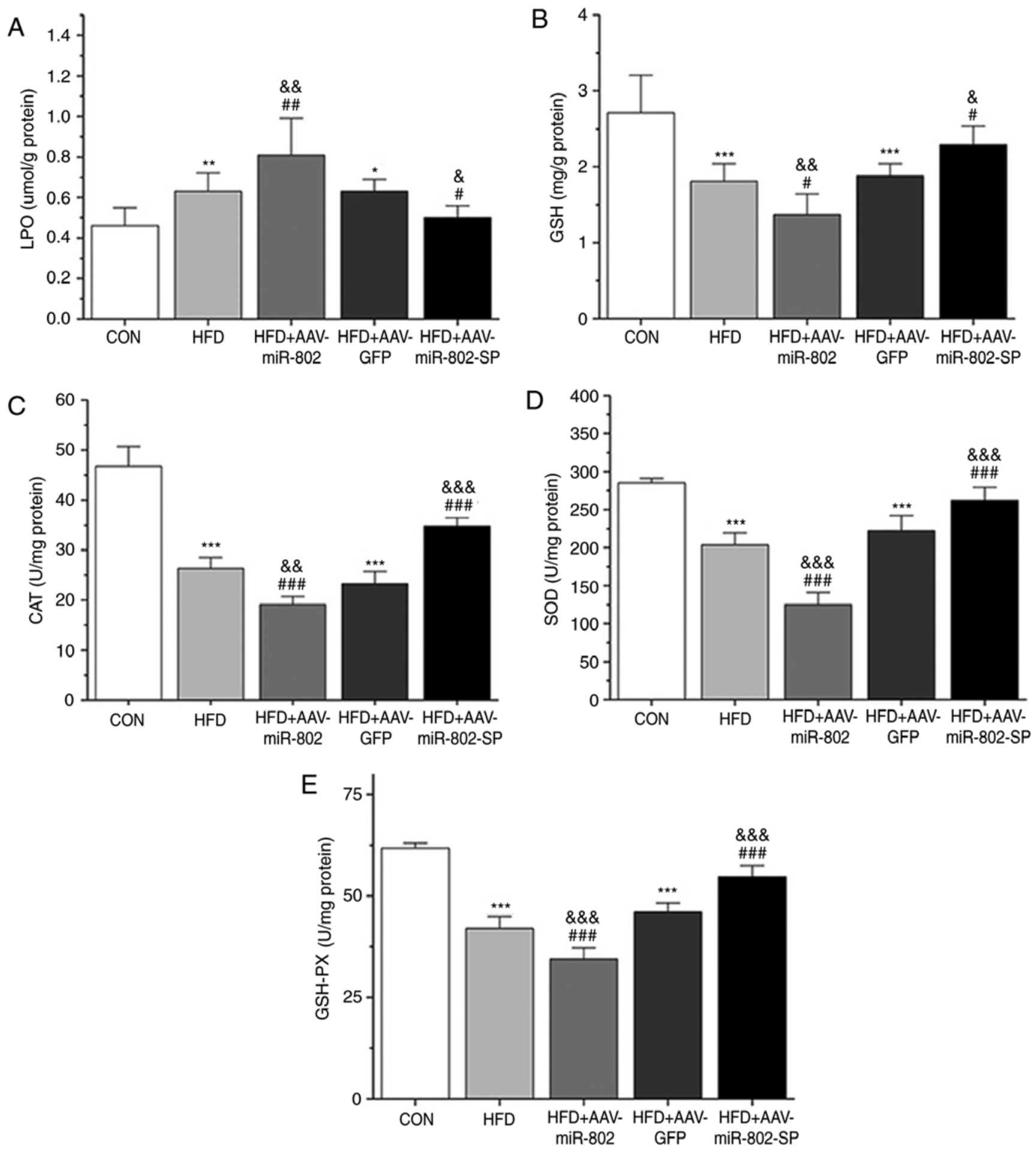

Figure 6. Effect of miR-802 on hepatic parameters. (A) LPO, (B) GSH levels and (C) CAT, (D) SOD, and (E) GSH-Px activity after 5 weeks of miR-802 treatment. Data are presented as the mean \pm standard error of the mean $(\mathrm{n}=6) .{ }^{*} \mathrm{P}<0.05,{ }^{* * *} \mathrm{P}<0.01$ and ${ }^{* * * *} \mathrm{P}<0.001$ vs. CON; ${ }^{\#} \mathrm{P}<0.05$, ${ }^{\# \#} \mathrm{P}<0.01$ and ${ }^{\# \# \#} \mathrm{P}<0.001$ vs. HFD; and ${ }^{\circledR} \mathrm{P}<0.05$, \&\& $<<0.01$ and ${ }^{\& \&} \mathrm{P}<0.001$ vs. HFD + AAV-GFP. miR-802, microRNA-802; LPO, lipid peroxidation; GSH, reduced glutathione; CAT, catalase; SOD, superoxide dismutase; GSH-Px, glutathione peroxidase; HFD, high-fat diet; CON, normal diet; AAV-GFP, adeno-associated virus vector containing green fluorescent protein; AAV-miR-802, AAVs expressing miR-802; AAV-miR-802-SP, AAVs expressing miR-802 sponges.

AKT1 were reduced compared with the AAV-GFP and HFD groups and the expression levels of phosphorylated IRS-1 (Ser307) were increased (Fig. 8). Notably, increased serine phosphorylation of IRS-1 reduces its phosphorylation in tyrosine residues, accelerates its degradation and subsequently reduces AKT phosphorylation (17). AAV-miR-802-SP completely reversed the effect of miR-802. These results suggest that miR-802 regulates the activity of individual components of the insulin signaling pathway.

Effect of miR-802 on the ERK, JNK and p38MAPK pathways in mouse liver. In order to investigate the downstream signal transduction mechanisms in IR induced by oxidative stress, the total and phosphor protein expression of ERK, JNK and 


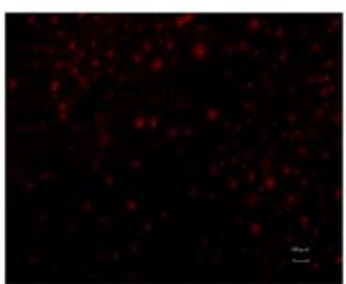

CON

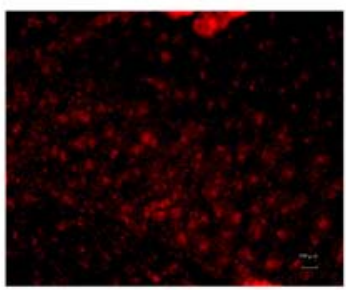

HFD+AAV-GFP

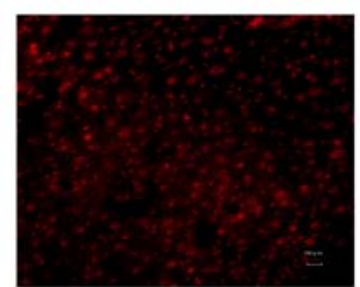

HFD

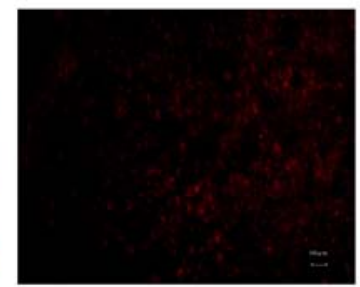

HFD+AAV-miR802-SP

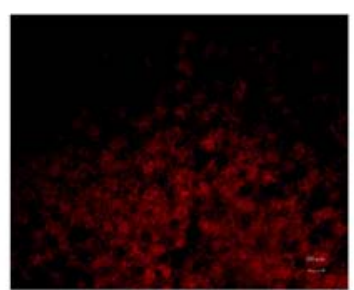

HFD+AAV-miR802

Figure 7. Effect of miR-802 on hepatic ROS generation as assessed by dihydroethidium. Original magnification, x100. miR-802, microRNA-802; ROS, reactive oxygen species; HFD, high-fat diet; CON, normal diet; AAV-GFP, adeno-associated virus vector containing green fluorescent protein; AAV-miR-802, AAVs expressing miR-802; AAV-miR-802-SP, AAVs expressing miR-802 sponges.

A
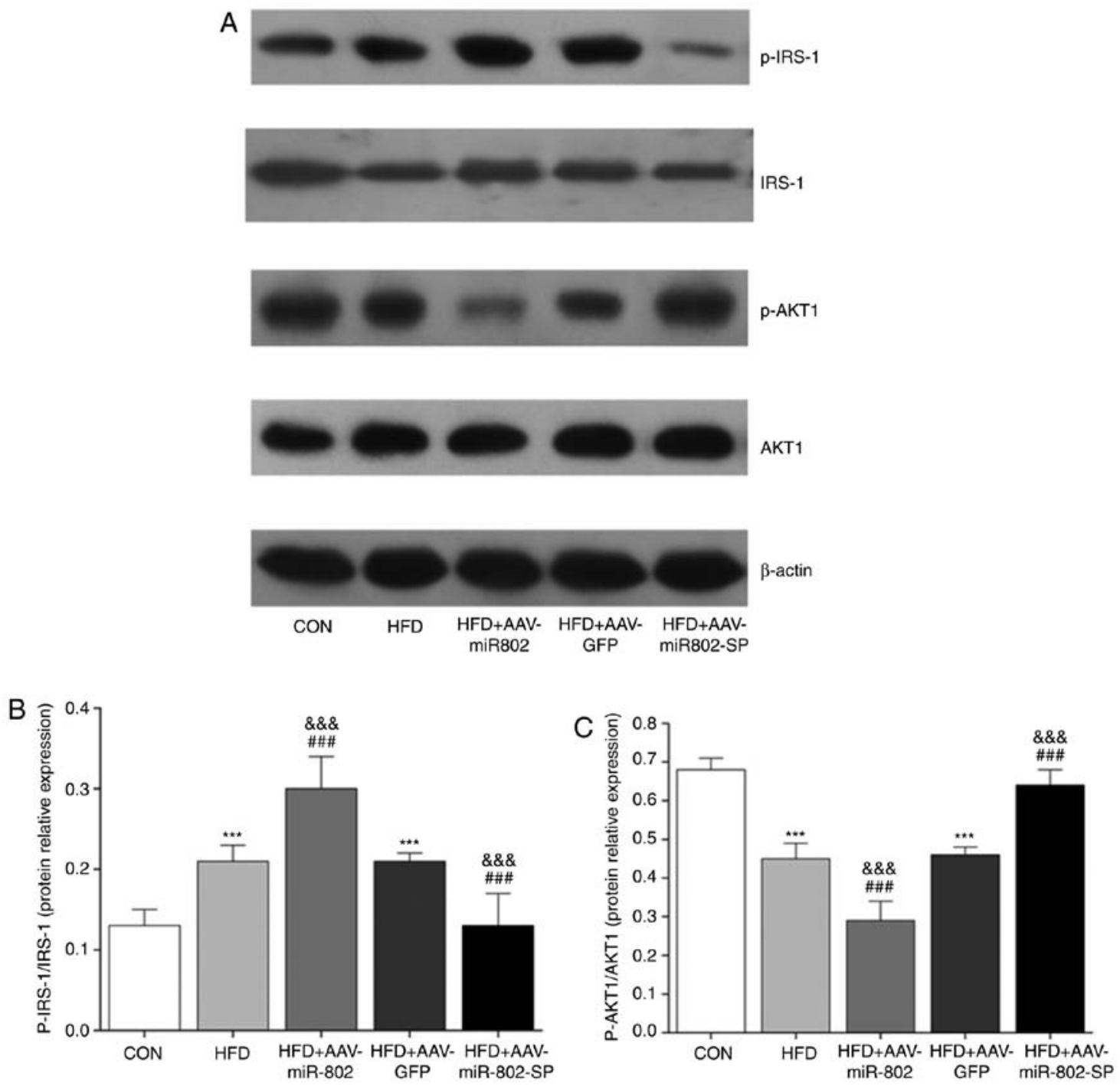

Figure 8. Effect of miR-802 on insulin pathways in the liver. (A) Western blot images. The protein expression levels of IRS-1, p-IRS-1, AKT1 and p-AKT1 were examined using western blotting. Densitometric analysis of the protein expression of (B) p-IRS-1/IRS-1 and (C) p-AKT/AKT. Data are presented as the mean \pm standard error of the mean $(n=6) .{ }^{* * *} \mathrm{P}<0.001$ vs. CON; ${ }^{* \#} \mathrm{P}<0.001$ vs. HFD; and ${ }^{\& \& \&} \mathrm{P}<0.001$ vs. HFD + AAV-GFP. miR-802, microRNA-802; CON, normal diet; HFD, high-fat diet; AAV-GFP, adeno-associated virus vector containing GFP; IRS-1, insulin receptor substrate 1; p-AKT1, phosphorylated protein kinase B; AAV-miR-802, AAVs expressing miR-802; AAV-miR-802-SP, AAVs expressing miR-802 sponges. 

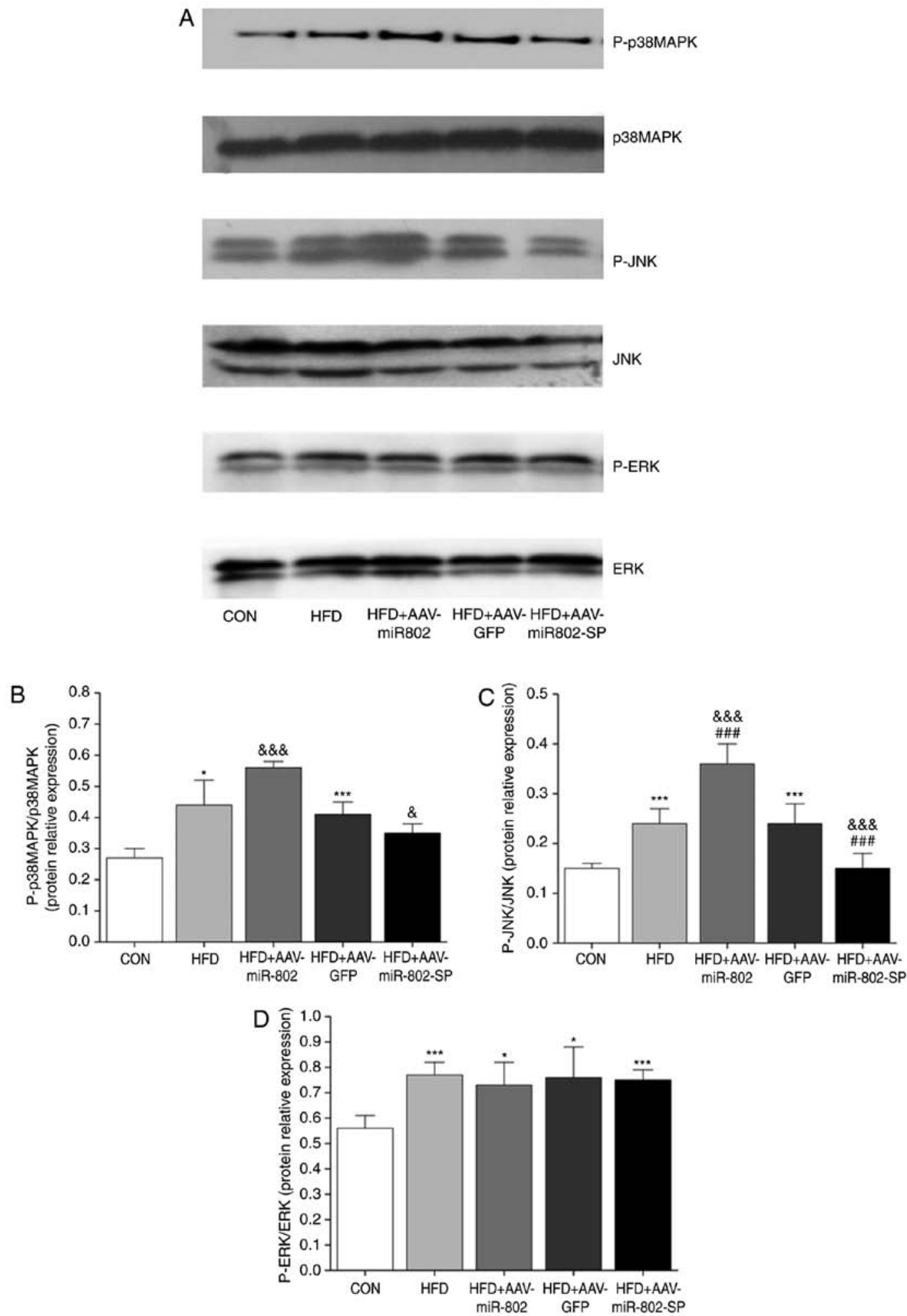

Figure 9. Effect of miR-802 on the ERK, JNK and p38MAPK pathways. (A) Western blot images. The protein expression levels of ERK, p-ERK, p38MAPK, p-p38MAPK, JNK and p-JNK were examined using western blotting. Densitometric analysis of the protein expression of (B) MAPK (C) JNK and (D) ERK. Data are presented as the mean \pm standard error of the mean $(n=6) .{ }^{*} \mathrm{P}<0.05$ and ${ }^{* * *} \mathrm{P}<0.001$ vs. CON; ${ }^{\# \# \#} \mathrm{P}<0.001$ vs. HFD; ${ }^{\&} \mathrm{P}<0.05$ and ${ }^{\& \& \&} \mathrm{P}<0.001$ vs. HFD + AAV-GFP. miR-802, microRNA-802; p38MAPK, p38mitogen-activated protein kinases; JNK, c-Jun N-terminal kinase; p-ERK, phosphorylated-extracellular signal regulated kinase; CON, normal diet; HFD, high-fat diet; AAV-GFP, adeno-associated virus vector containing green fluorescent protein; AAV-miR-802, AAVs expressing miR-802; AAV-miR-802-SP, AAVs expressing miR-802 sponges.

p38MAPK in the liver tissue of the mice were determined after 5 weeks of miR-802 treatment. Only the p38MAPK and JNK pathways were activated, as demonstrated by increased phosphorylation of p38MAPK and JNK. However, the phosphorylation of ERK was not significantly different (Fig. 9), suggesting that miR-802 may have no effect on ERK activity. These results suggest that miR-802 activates the p38MAPK and JNK signaling pathways, but not that of ERK. 


\section{Discussion}

The present study identifies a novel miRNA-dependent mechanism of IR. The high-fat-induced model of IR is more consistent with the pathogenesis of diabetes in humans than $\mathrm{db} / \mathrm{db}$ or ob/ob mice (18). As a classical model of IR, the high-fat induced IR model has been used for a long time in the authors' laboratory and therefore they have had enough experience to use this model successfully. The laboratory provides sufficient conditions for model establishment.

In the present study, the effect of miR-802 on HFD-induced oxidative stress in the liver was investigated and the results demonstrate that miR-802 overexpression results in increased oxidative stress in mouse livers. Notably, miR-802 overexpression further aggravated hyperinsulinemia, hyperglycemia and oxidative stress in the HFD fed mice. This effect may be caused by activation of the p38MAPK and JNK pathways. These results suggest that increased miR-802 expression is one of the main causes of obesity-associated metabolic abnormalities. The causative role of miR-802 was further confirmed by silencing miR-802 in the mice, which improved insulin sensitivity and glucose homeostasis, and reduced oxidative stress. These results suggest that downregulation of miR-802 is sufficient to prevent progression of obesity-associated metabolic syndrome.

Hepatic lipid accumulation has emerged as one of the major culprits in the pathogenesis of T2DM (19). In the present study, miR-802 had no effect on body weight or serum TG and TC. Professor Kornfeld (8) found that insulin sensitivity was improved and glucose intake increased, but weight didn't change, after the expression of miR-802 was knocked down in HFD mice. Results of the present study are consistent with the Professor Kornfeld's study, however, the mechanism remains to be fully elucidated. The possible reasons: i) The intervening time was not enough to influence the weight of the mice. In subsequent experiments, the observation time will be extended to further explore the effect of miR802 on body weight in HFD mice; ii) miR-802 improved insulin resistance may occur by increasing the uptake of glucose in tissues and organs but not by affecting fat content.

Conversely, the levels of hepatic TG were increased after miR-802 treatment and miR-802-SP reduced the levels of hepatic TG in the HFD fed mice. Therefore, miR- 802 is an important regulator of HFD-induced hyperlipidemia in the liver.

It has been reported that miR-802 targets $\mathrm{Hnf} 1 \mathrm{~b}$ in the livers of HFD fed and Lepr ${ }^{\mathrm{db} / \mathrm{db}}$ mice, leading to IR (8). Hnflb has been causally linked to the development of maturity-onset diabetes of the young type 5 and variants in HNF1B have been linked to predisposition for T2DM (20). However, it is unclear whether miR-802 serves a role in regulating oxidative stress in HFD fed mice.

It has been reported that consumption of a HFD can significantly increase oxidative stress (21). Ectopic overload of fatty acids has been proposed as a cause of type 2 diabetes, through accelerating excess generation of ROS. The results of the presents study revealed increased levels of oxidative stress in the livers of the HFD fed mice, which is consistent with previous studies (22-24). The current study demonstrated that miR-802 further increases the levels of oxidative stress in the livers of HFD fed mice, which is likely to be caused by reduced activity of multiple antioxidative enzymes.
Chronic oxidative stress that is mainly caused by mitochondrial dysfunction can lead to the development T2DM and metabolic syndrome (25). ROS directly oxidize and damage DNA, proteins and lipids. In addition to their ability to directly inflict damage on macromolecules, ROS indirectly induce damage to tissues by activating a number of cellular stress-sensitive pathways, including the JNK (26), p38MAPK and ERK1/2 pathways (27).

A previous study revealed that the family members of MAPK, ERK1/2, JNK and p38MAPK potentially contribute to the abnormal insulin signaling associated with T2DM (28). A further study demonstrated that stress activation of JNK/SAPK increases the levels of serine phosphorylation (at Ser307) and inhibits insulin-stimulated tyrosine phosphorylation of IRS-1 (29). The results of the present study indicate that a HFD promotes the phosphorylation of JNK and p38MAPK. This may lead to further IRS-1 phosphorylation and less AKT1 phosphorylation, which impairs insulin signaling.

The effect of oxidative stress on cellular signaling pathways and activation of the JNK and p38MAPK pathways was enhanced by miR-802. The insulin-signaling pathway is the central part of the action of insulin. The present study further identified that miR-802 reduces the activity of the insulin signaling pathway, therefore enhancing the IR of HFD fed mice. The decreased activity of insulin signaling molecules may be a result of the increased activities of JNK and p38MAPK caused by miR-802. Dual-luciferase assays, miRNA microarrays and western blotting should be used to confirm the potential target genes of miR-802 in skeletal muscle in future studies in order to identify whether the effect is direct or indirect. Furthermore, it may be of interest to further investigate whether miR-802 could be used as a biomarker for the diagnosis of diabetes.

The present study demonstrates that miR-802 induces IR in HFD fed mice by increasing hepatic oxidative stress. miR-802 achieves this through upregulation of the p38MAPK and JNK pathways and downregulation of the insulin signaling pathway. Although the detailed mechanisms regarding how miR-802 regulates liver IR require further investigation, the results of the present study provided a mechanistic basis for the targeting of miR-802 as a potential therapeutic strategy to combat oxidative stress and IR.

\section{Acknowledgements}

The present study was supported in part by the Hebei Key Laboratory of Metabolic Disease, Hebei General Hospital. The authors would like to thank Dr Linlin Yang and Dr Donghui Zhang for their technical assistance.

\section{Funding}

The present study was supported by the International cooperation Program of Hebei Province (grant no. 15397750d) and the National Natural Science Foundations of China (grant no. 81200638).

\section{Availability of data and materials}

The datasets used and/or analyzed during the current study are available from the corresponding author on reasonable request. 


\section{Authors' contribution}

XY collected and analyzed the majority of the data and wrote the manuscript. HX collected and analyzed data and edited the manuscript. JL, LY and HuaM performed the experiments. HuiM was involved in study design, oversaw data collection and analysis and wrote, and edited the manuscript.

\section{Ethics approval and consent to participate}

All experiments involving animals were performed according to the procedures approved by the Institutional Animal care and Use committee of the Institute of Zoology, Hebei General Hospital (Shijiazhuang, China).

\section{Patient consent for publication}

Not applicable.

\section{Competing interests}

The authors declare they have no actual or potential competing financial interests.

\section{References}

1. Lee BC and Lee J: Cellular and molecular players in adipose tissue inflammation in the development of obesity-induced insulin resistance. Biochim Biophys Acta 1842: 446-462, 2014.

2. Dehwah MA, Xu A and Huang Q: MicroRNAs and type 2 diabetes/obesity. J Genet Genomics 39: 11-18, 2012.

3. Bartel DP: MicroRNAs: Target recognition and regulatory functions. Cell 136: 215-233, 2009.

4. Trajkovski M, Hausser J, Soutschek J, Bhat B, Akin A, Zavolan M, Heim MH and Stoffel M: MicroRNAs 103 and 107 regulate insulin sensitivity. Nature 474: 649-653, 2011.

5. Zhou B, Li C, Qi W, Zhang Y, Zhang F, Wu JX, Hu YN, Wu DM, Liu Y, Yan TT, et al: Downregulation of miR-181a upregulates sirtuin-1 (SIRT1) and improves hepatic insulin sensitivity. Diabetologia 55: 2032-2043, 2012.

6. Fu X, Dong B, Tian Y, Lefebvre P, Meng Z, Wang X, Pattou F, Han W, Wang X, Lou F, et al: MicroRNA-26a regulates insulin sensitivity and metabolism of glucose and lipids. J Clin Invest 125: 2497-2509, 2015.

7. Higuchi C, Nakatsuka A, Eguchi J, Teshigawara S, Kanzaki M, Katayama A, Yamaguchi S, Takahashi N, Murakami K, Ogawa D, et al: Identification of circulating miR-101, miR-375 and miR-802 as biomarkers for type 2 diabetes. Metabolism 64: 489-497, 2015

8. Kornfeld JW, Baitzel C, Konner AC, Nicholls HT, Vogt MC, Herrmanns K, Scheja L, Haumaitre C, Wolf AM, Knippschild U, et al: Obesity-induced overexpression of miR-802 impairs glucose metabolism through silencing of Hnf1b. Nature 494: 111-115, 2013.

9. Matsuzawa-Nagata N, Takamura T, Ando H, Nakamura S, Kurita S, Misu H, Ota T, Yokoyama M, Honda M, Miyamoto K and Kaneko S: Increased oxidative stress precedes the onset of high-fat diet-induced insulin resistance and obesity. Metabolism 57: 1071-1077, 2008

10. Evans JL, Maddux BA and Goldfine ID: The molecular basis for oxidative stress-induced insulin resistance. Antioxid Redox Signal 7: 1040-1052, 2005.

11. Manno CS, Pierce GF, Arruda VR, Glader B, Ragni M, Rasko JJ, Ozelo MC, Hoots K, Blatt P, Konkle B, et al: Successful transduction of liver in hemophilia by AAV-Factor IX and limitations imposed by the host immune response. Nat Med 12: 342-347, 2006.
12. Grimm D and Kay MA: Therapeutic short hairpin RNA expression in the liver: Viral targets and vectors. Gene Ther 13: 563-575, 2006.

13. Bagul PK, Middela H, Matapally S, Padiya R, Bastia T, Madhusudana K, Reddy BR, Chakravarty S and Banerjee SK: Attenuation of insulin resistance, metabolic syndrome and hepatic oxidative stress by resveratrol in fructose-fed rats. Pharmacol Res 66: 260-268, 2012.

14. Katz A, Nambi SS, Mather K, Baron AD, Follmann DA, Sullivan G and Quon MJ: Quantitative insulin sensitivity check index: A simple, accurate method for assessing insulin sensitivity in humans. J Clin Endocrinol Metab 85: 2402-2410, 2000.

15. Benov L, Sztejnberg L and Fridovich I: Critical evaluation of the use of hydroethidine as a measure of superoxide anion radical. Free Radic Biol Med 25: 826-831, 1998.

16. Das M, Das S, Lekli I and Das DK: Caveolin induces cardioprotection through epigenetic regulation. J Cell Mol Med 16: 888-895, 2012

17. Pessin JE and Saltiel AR: Signaling pathways in insulin action: Molecular targets of insulin resistance. J Clin Invest 106: $165-169,2000$.

18. Sone H, Takahashi A, Iida K and Yamada N: Disease model Hyperinsulinemia and insulin resistance. Part B-polygenic and other animal models. Trends Mol Med 7: 373-376, 2001.

19. Perry CG and Wright DC: Challenging dogma: Is hepatic lipid accumulation in type 2 diabetes due to mitochondrial dysfunction? J Physiol 594: 4093-4094, 2016.

20. Han X, Luo Y, Ren Q, Zhang X, Wang F, Sun X, Zhou X and Ji L: Implication of genetic variants near SLC30A8, HHEX, CDKAL1, CDKN2A/B, IGF2BP2, FTO, TCF2, KCNQ1, and WFS1 in type 2 diabetes in a Chinese population. Bmc Med Genet 11: 81, 2010.

21. Erdemir F, Atilgan D, Markoc F, Boztepe O, Suha-Parlaktas B and Sahin S: The effect of diet induced obesity on testicular tissue and serum oxidative stress parameters. Actas Urol Esp (Spanish) 36: 153-159, 2012.

22. Gujjala S, Putakala M, Gangarapu V, Nukala S, Bellamkonda R, Ramaswamy R and Desireddy S: Protective effect of Caralluma fimbriata against high-fat diet induced testicular oxidative stress in rats. Biomed Pharmacother 83: 167-176, 2016.

23. Feng X, Yu W, Li X, Zhou F, Zhang W, Shen Q, Li J, Zhang C and Shen P: Apigenin, a modulator of PPAR $\gamma$, attenuates HFD-induced NAFLD by regulating hepatocyte lipid metabolism and oxidative stress via Nrf2 activation. Biochem Pharmacol 136: 136-149, 2017.

24. Pan ZG and An XS: SARM1 deletion restrains NAFLD induced by high fat diet (HFD) through reducing inflammation, oxidative stress and lipid accumulation. Biochem Biophys Res Commun 498: 416-423, 2018.

25. Styskal J, Van Remmen H, Richardson A and Salmon AB: Oxidative stress and diabetes: What can we learn about insulin resistance from antioxidant mutant mouse models? Free Radic Biol Med 52: 46-58, 2012

26. Zhang ZF, Lu J, Zheng YL, Wu DM, Hu B, Shan Q, Cheng W, Li MQ and Sun YY: Purple sweet potato color attenuates hepatic insulin resistance via blocking oxidative stress and endoplasmic reticulum stress in high-fat-diet-treated mice. J Nutr Biochem 24: 1008-1018, 2013.

27. Sankar P, Zachariah B, Vickneshwaran V, Jacob SE and Sridhar MG: Amelioration of oxidative stress and insulin resistance by soy isoflavones (from Glycine max) in ovariectomized Wistar rats fed with high fat diet: The molecular mechanisms. Exp Gerontol 63: 67-75, 2015.

28. Carlson CJ, Koterski S, Sciotti RJ, Poccard GB and Rondinone CM: Enhanced basal activation of mitogen-activated protein kinases in adipocytes from type 2 diabetes: Potential role of p38 in the downregulation of GLUT4 expression. Diabetes 52: 634-341, 2003.

29. Bloch-Damti A and Bashan N: Proposed mechanisms for the induction of insulin resistance by oxidative stress. Antioxid Redox Signal 7: 1553-1567, 2005.

This work is licensed under a Creative Commons Attribution-NonCommercial-NoDerivatives 4.0 International (CC BY-NC-ND 4.0) License. 